\title{
Alveolar Soft Part Sarcoma: Should We Be Targeting the Tumor or Targeting the Vasculature?
}

\author{
Jaap Verweij \\ Department of Medical Oncology, Erasmus University Medical Center - Daniel den Hoed Cancer Center, Rotterdam, The Netherlands
}

Soft tissue sarcomas constitute a heterogeneous group of tumors that, due to their rare occurrence, used to be pooled for treatment assessment purposes. With the improvement in diagnostics and the development of molecularly targeted agents one has become increasingly aware that it is important, however, to differentiate the various subtypes from one another [1]. The development of imatinib for gastrointestinal stromal tumors (GIST) [2] can serve as an example. Alveolar soft part sarcoma (ASPS) is an extremely rare subtype of soft tissue sarcoma. It is characterized by translocation der(17) $\mathrm{t}(\mathrm{X}: 17)(\mathrm{p} 11 ; \mathrm{p} 25)$ on one hand, and a usually indolent growth pattern on the other [3]. Similar to GIST, it is quite insensitive to the commonly used cytotoxic drugs [4].

Trabectedin (ET-743; Yondelis ${ }^{\circledR}$, PharmaMar SA, Madrid, Spain), an agent isolated from the Caribbean tunicate Ecteinascidia turbinata. Trabectedin is a cytotoxic drug that binds to the minor groove of DNA [5], and induces double-stranded DNA breaks [6] and aG2-M cell cycle arrest [7]. Trabectedin is registered for use in soft tissue sarcomas in the EU and several other countries. While in general the response rates observed with its use are low, the level of progression arrest achieved is considerable [8]. However, studies on the activity of the agent in ASPS are lacking. In this issue of ONKOLOGIE, Pink et al. [9] present a retrospective analysis on a collected case series of 7 patients with ASPS treated with trabectedin. The retrospective collection and analysis of the series is hampered by the inevitable limitations of each retrospective analysis, for instance the impossibility to exclude an unintended patient selection bias. This in itself calls for major caution in trying to interpret the data of any case report based series.

Five of the 7 patients were treated with the registered dose for trabectedin, which is $1.5 \mathrm{mg} / \mathrm{m}^{2}$ as a $24-\mathrm{h}$ continuous intravenous infusion, repeated every 21 days. 2 patients were given a reduced dose. A clear dose-response relationship for trabectedin in soft tissue sarcoma has not been described yet, but if there is one, it is unlikely this will have negatively influenced the outcome interpretation of the treatment in the cur- rent series. On the other hand 1 or 2 of the patients had not been pretreated with chemotherapy. This aspect can be of influence since there are data to suggest differences in the outcome of trabectedin treatment, depending on the level of pretreatment with cytotoxics [8].

However, all of these are minor issues to put the observations into context. The most important issue is the absence of formal response evaluation criteria in solid tumors (RECIST)based tumor regressions in the patients treated. This renders it important to try and assess the relevance of stable disease (SD). Acknowledging the potential relevance of SD in patients with soft tissue sarcomas the European Organization for Treatment and Research of Cancer (EORTC) Soft Tissue and Bone Sarcoma Group has propagated the use of progression free rates (PFR) as a tool for clinical trial purposes in screening studies to assess the potential activity of the drug under study [10]. The 6-month PFR of 57\% reported by Pink et al. [9] is clearly higher than the $40 \%$ 6-month PFR indicated by the EORTC to suggest an agent may be active. However, ASPS patients were not included in the EORTC studies that were used to create the reference. Due to the indolent behavior of this subtype it is quite likely that the PFR boundaries indicated by the EORTC cannot be applied to ASPS, since they would lead to overestimation of actual drug activity. So how can we be sure that the suggested activity is relevant? The other way to assess this would be by use of the growth modulation index (GMI) [11], as the investigators have done, comparing the time to progression (TTP) on the current treatment to the TTP on the previous treatment. This can only be done in patients that have been pretreated. In this respect there seems to be a possible inconsistency in the paper concerning patient $\mathrm{F}$ that in table 1 is listed as pretreated and in table 2 as non-pretreated. If the latter where correct, the GMI cannot be calculated and thus not reported in table 3 for patient F. However, even when excluding this patient, in all pretreated patients the GMI this seems to favor the trabectedin use. Again, a word of caution is indicated, since one has to realize the data on TTP on the prior therapy, due to the retro-

\section{KARGER \\ Fax +497614520714 \\ Information@Karger.de}

www.karger.com (c) 2012 S. Karger GmbH, Freiburg

0378-584X/12/0355-0237\$38.00/0

Accessible online at:

www.karger.com/onk
Jaap Verweij, MD, PhD, Medical Oncologist

Department of Medical Oncology

Erasmus University Medical Center - Daniel den Hoed Cancer Center

's-Gravendijkwal 230, 3015 CE Rotterdam, The Netherlands

Tel. + 3110 7034-447, Fax -627

j.verweij@erasmusmc.nl 
spective nature of the analysis, could not be collected in a standardized way. Therefore, even with the details provided in the table in the paper of Pink et al. [9], it remains impossible for the reader to fully assess the relevance. One may state that for ASPS the TTP of 2-5 months on previous therapy in the reported patients is relatively short for an indolent disease such as ASPS. This could reflect a relatively aggressive behavior of the disease in the patients treated, but could also reflect ineffectivity of the prior treatment used. Most importantly, without seeing actual measurement data, an interpretation of the observed GMI remains difficult.

If we take the disease stabilization rate reported by the authors as $86 \%$, it is important to realize that the $95 \%$ confidence limits of the observation still vary from 42 to $99 \%$ and that the chance of a false positive observation remains $7-58 \%$.

This is one of the two reasons why one has to remain careful in taking the statements of the authors that 'trabectedin can be considered the only currently registered drug with clinical activity in this disease' and '...trabectedin can be considered as a fairly well tolerated therapy option to achieve disease stabilization in patients who had experienced disease progression'. The second reason is that there may be other and possibly better options for these patients, as partly correctly referred to by Pink et al. [9] as well. In general the vascular endothelial growth factor (VEGF)-based multi-tyrosine kinase inhibitors seem to exert important activity in ASPS [12-14]. Initially similar collected case series as the one from
Pink et al. [9] suggested activity including a relevant number of RECIST based partial remissions with cediranib [12] and sunitinib [13]. The latter drug is commercially available and could thus be considered a treatment option. Importantly the case report observations have been confirmed in a formal phase II study on cediranib [14]. Kummar et al. [14] studied 36 patients, and at the time of reporting could evaluate 28 for response. 12 of these patients achieved a RECIST PR (43\%), 4 had $>20 \%$ reduction in lesion size, and 6 additional ones were stable for at least 6 months. This represents a disease control rate at 6 months of $78 \%$. Importantly many of these disease controls were already known to last much longer than 6 months. While cediranib is no longer available, these data seem to suggest that VEGFR inhibitors may have major activity in ASPS. While study treatment still would be preferred and direct comparison between agents is lacking, consideration of this class of agents for patients with ASPS would be worthwhile.

The observations from Pink et al. [9] are certainly interesting, but should be viewed with caution and should be placed into appropriate perspective.

\section{Disclosure Statement}

The author declares no conflict of interest.

\section{References}

1 Verweij J: Soft tissue sarcoma trials: one size no longer fits all. J Clin Oncol 2009;27:3085-3087.

2 Verweij J, Casali P, Zalcberg J, Le Cesne A, Reichardt P, Blay J-Y, Issels R, van Oosterom A Hogendoorn P, van Glabbeke M, Judson I, Bertulli R, Judson I, for the EORTC Soft Tissue and Bone Sarcoma Group, the Italian Sarcoma Group and the Australian Gastro-intestinal trials group: Progression-free survival in gastrointestinal stromal tumours with high-dose imatinib: randomised trial. Lancet 2004; 364:1127-1134

3 Pennacchioli E, Fiore M, Collini P, Radaelli S, Dileo P, Stacchiotti S, Casali PG, Gronchi A: Alveolar soft part sarcoma: clinical presentation, treatment, and outcome in a series of 33 patients at a single institution. Ann Surg Oncol 2010;17:3229_ 3233.

4 Scurr M: Histology-driven chemotherapy in soft tissue sarcomas. Curr Treat Options Oncol 2011; 12:32-45.

5 Pommier Y, Kohlhagen G, Bailly C, Waring M, Mazumder A, Kohn KW: DNA sequence- and structure-selective alkylation of guanine N2 in the DNA minorgroove by ecteinascidin 743 , a potent antitumor compound from the Caribbean tunicate Ecteinascidia turbinata. Biochemistry 1996;35: 13303-13309.
6 Takebayashi Y, Pourquier P, Zimonjic DB, Nakayama K, Emmert S, Ueda T, Urasaki Y, Kanzaki A, Akiyama SI, Popescu N, Kraemer KH, Pommier Y: Antiproliferative activity of ecteinascidin 743 is dependent upon transcriptioncoupled nucleotide-excision repair. Nat Med 2001;7:961966.

7 Gajate C, An F, Mollinedo F: Differential cytostatic and apoptotic effects of ecteinascidin-743 in cancer cells. Transcription-dependent cell cycle arrest and transcription-independent JNK and mitochondrial mediated apoptosis. J Biol Chem 2002;277:41580-41589.

8 Verweij J: Ecteinascidin-743 (ET-743): early test or effective treatment in soft tissue sarcomas? J Clin Oncol. 2005;23:5420-3

9 Pink D, Bertz-Lepel J, Busemann Ch, Bitz U, Reichardt P: Efficacy of trabectedin in patients with advanced or metastatic alveolar soft part sarcoma (ASPS). Onkologie 2012:35: DOI: $10.1159 / 000338342$.

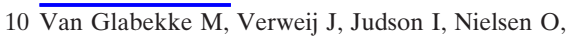
on behalf of the EORTC Soft Tissue and Bone Sarcoma Group: Progression-free rate as the principal end-point for phase II trials in soft-tissue sarcoma. Eur J Cancer 2002;38:543-549.
11 Verweij J: Other endpoints in screening studies for soft tissue sarcomas. Oncologist 2008;13(suppl 2):27-31.

12 Gardner K, et al.: Activity of cediranib, a highly potent and selective VEGF signaling inhibitor, in alveolar soft part sarcoma. J Clin Oncol 2009;27:15s (suppl, abstr 10523)

13 Palassini E, Stacchiotti S, Negri T, Brich S, Marrari A, Morosi C, Crippa F, Gronchi A, Pilotti S, Casali PG: Sunitinib malate (SM) in alveolar soft part sarcoma (ASPS). J Clin Oncol 2010; 28:15s (suppl, abstr 10014).

14 Kummar S, Strassberger A, Monks A, Ivy SP, Turkbey IB, Choyke PL, Steinberg SM, Simon R, Doroshow JH, Helman LJ: An evaluation of cediranib as a new agent for alveolar soft part sarcoma (ASPS). J Clin Oncol 2011;29:(suppl; abstr 10001). 